\title{
A note on uniqueness of entropy solutions to degenerate parabolic equations in $\mathbb{R}^{N}$
}

\author{
Boris Andreianov and Mohamed Maliki
}

\begin{abstract}
We study the Cauchy problem in $\mathbb{R}^{N}$ for the parabolic equation

$$
u_{t}+\operatorname{div} F(u)=\Delta \varphi(u),
$$

which can degenerate into a hyperbolic equation for some intervals of values of $u$. In the context of conservation laws (the case $\varphi \equiv 0$ ), it is known that an entropy solution can be non-unique when $F^{\prime}$ has singularities. We show the uniqueness of an entropy solution to the general parabolic problem for all $L^{\infty}$ initial datum, under the isotropic condition on the flux $F$ known for conservation laws. The only assumption on the diffusion term is that $\varphi$ is a non-decreasing continuous function.
\end{abstract}

Mathematics Subject Classification (2000). Primary 35K65; Secondary 35A05.

Keywords. Degenerate hyperbolic-parabolic equation - Conservation law · Non-Lipschitz flux · Entropy solution · Kato inequality · Infinite speed of propagation · Uniqueness.

\section{Contents}

1. Introduction

2. Definitions and results 112

3. Proof of Theorem 2 114

$\begin{array}{ll}\text { References } & 117\end{array}$

The referees' careful reading and remarks improved the paper and simplified the assumptions initially made by the authors.

B. Andreianov acknowledges the hospitality of the FST Mohammédia, of the CMA Oslo, and of IZKS, Bonn University (supported by the DFG project 436 RUS 113/895/0-1). 


\section{Introduction}

Consider the convection-diffusion problem

$$
u_{t}+\operatorname{div} F(u)-\Delta \varphi(u)=f \quad \text { in }(0, T) \times \mathbb{R}^{N},\left.\quad u\right|_{t=0}=u_{0}
$$

with a source $f$ measurable on $(0, T) \times \mathbb{R}^{N}$, with $\int_{0}^{T}\|f(t, \cdot)\|_{L^{\infty}} d t<+\infty$ and an initial datum $u_{0} \in L^{\infty}\left(\mathbb{R}^{N}\right)$. Here $F=\left(F_{1}, \ldots, F_{N}\right): \mathbb{R} \mapsto \mathbb{R}^{N}$ is assumed merely continuous (a quantitative continuity assumption on $F$ will be imposed in the sequel), and $\varphi: \mathbb{R} \mapsto \mathbb{R}$ is a continuous non-decreasing function.

Degenerate and non-degenerate problems of the kind (1), often investigated under the local Lipschitz continuity assumption on $\varphi$ and $F$, appear in various physical contexts, including e.g. flows and sedimentation in porous media. Mathematical treatment of these problems was boosted some ten years ago by the introduction of the adequate notion of entropy solution (see Carrillo [8]).

Indeed, the framework (1) includes conservation laws as a particular case. It is well known that in general, global classical solutions of (1) may not exist; and that a weak solution in the sense of distributions may not be unique. The standard way to fix this problem is to work with the so-called entropy solutions (see Kruzhkov [9] for the case of conservation laws, and Carrillo [8] for the adaptation of this notion to the case of a degenerate elliptic-parabolichyperbolic equation, of which (1) is a particular case). The classical result of Kruzhkov establishes uniqueness of an entropy solution (together with the property of finite domain of dependence on initial data) for the case where $\varphi \equiv 0$ and $F$ is locally Lipschitz continuous. When the Lipschitz continuity assumption is dropped, uniqueness of entropy solutions for a conservation law can be lost (see the works $[10,13,14,16]$ of Kruzhkov and Panov). The effect of non-uniqueness is closely connected to the property of "infinite speed of propagation", which arises when $F^{\prime}$ is unbounded.

This is the goal of this note to investigate the question of uniqueness of entropy solutions of (1) for a general nonlinearity $\varphi$ in the diffusion term. Technically, the problem amounts to the difficulty of exploiting the so-called Kato inequality

$$
\begin{aligned}
& (u-\hat{u})_{t}^{+}+\operatorname{div}\left(\operatorname{sign}^{+}(u-\hat{u})(F(u)-F(\hat{u}))\right. \\
& \quad \leq \Delta(\varphi(u)-\varphi(\hat{u}))^{+}+(f-\hat{f})^{+} \quad \text { in } \mathcal{D}^{\prime}\left([0, T) \times \mathbb{R}^{N}\right)
\end{aligned}
$$

here $u, \hat{u}$ are two entropy solutions of (1) corresponding to source terms $f, \hat{f}$. Formally, if we let the test function in (2) converge to one, we get the inequality

$$
\frac{d}{d t} \int_{\mathbb{R}^{N}}(u-\hat{u})^{+}(t) \leq \int_{\mathbb{R}^{N}}(f-\hat{f})^{+} \quad \text { in } \mathcal{D}^{\prime}([0, T)),
$$

which implies uniqueness of an entropy solution. Even when one can justify that $u-\hat{u} \in L^{\infty}\left(0, T ; L^{1}\left(\mathbb{R}^{N}\right)\right)$, a too slow decrease of $|F(u)-F(\hat{u})|$ as $|x| \rightarrow \infty$ could be an obstacle to this formal reasoning. In fact, the issue of uniqueness of $L^{1} \cap L^{\infty}$ solutions for the conservation law $u_{t}+\operatorname{div} F(u)=0,\left.u\right|_{t=0}=u_{0}$ is not yet clearly understood; see [2] for a discussion of relevancy of the known conditions. 
For the case $\varphi \equiv 0$ and $L^{\infty}$ entropy solutions, sufficient uniqueness conditions for (1) in terms of the moduli of continuity of $F$ were first formulated by Kruzhkov and Hil'debrand [12] (after that Bénilan [5] obtained the same condition for mild solutions in $L^{1}$ ). Roughly speaking, the conditions of [5,12] (see also [11]) amount to the Hölder continuity of $F$ of order $\alpha=1-\frac{1}{N}$. This condition is "isotropic": the possibility that some of the components $F_{1}, \ldots, F_{N}$ of $F$ may have a "better" modulus of continuity than the vector-function $F$ is not exploited. This contrasts with the following "anisotropic" assumption

$$
\liminf _{r \downarrow 0} \frac{\prod_{i=1}^{N} \omega_{i}(r)}{r^{N-1}}<+\infty
$$

due to Kruzhkov and Panov $[13,16]$; here for $i=1, \ldots, N, \omega_{i}: \mathbb{R}^{+} \rightarrow \mathbb{R}^{+}$ is a modulus of continuity of $F_{i}$ on the interval $[-M, M]$ containing the values of $u$. Assumption (3) restricts the behaviour of the moduli of continuity $\omega_{i}(r)$ of $F_{i}$ at $r=0$. Assumption (3) is also sufficient for the uniqueness of entropy solutions of a scalar conservation law. A sharper condition is given in [14]. Notice that for the $L^{1} \cap L^{\infty}$ data, sufficient uniqueness conditions of a completely different nature were found by Bénilan and Kruzhkov in [6] (see also [2]).

In [15], together with Touré the second author of the present paper proved uniqueness of an entropy solution to Eq. (1) upon replacing $\omega_{i}(r)$ in (3) with $\left(\omega_{i}(r)+\left(r \omega_{\varphi}(r)\right)^{1 / 2}\right)$; here $\omega_{\varphi}: \mathbb{R}^{+} \rightarrow \mathbb{R}^{+}$is a modulus of continuity of $\varphi$. Clearly, if the space dimension $N$ equals 1 , both the Kruzhkov-Panov and the Maliki-Touré conditions are always satisfied. If the space dimension is equal to 2 and the isotropic case $\omega_{1}=\omega_{2}$ is considered, then the Maliki-Touré condition contains no restriction on the function $\varphi$.

In the work [3] we have investigated the exactness of the assumption of [15] for the case of a pure diffusion equation (i.e., $F \equiv 0$ ). It turns out that uniqueness of $L^{\infty}$ solutions for this case holds for all $\varphi$. In the present paper, we show a similar result for the full convection-diffusion equation (1). More exactly, we show uniqueness of an entropy solution for (1) for all continuous non-decreasing $\varphi$, but under the assumption that $F$ satisfies the isotropic local Hölder continuity condition of order $\alpha=1-\frac{1}{N}$. According to the previous remark, we can restrict our attention to the case $N \geq 3$.

Let us briefly describe the main idea of the proof. In order to make disappear the third term in (2), we choose a test function $\rho$ that is, roughly speaking, a truncation of the fundamental solution to the Laplace equation in $\mathbb{R}^{N}$. Notice that we cannot avoid taking $\rho$ convex in the radial direction, because we need $\rho$ to be non-negative and equal to zero at infinity. Yet we can take $\rho$ concave in the orthogonal directions, so that compensations make non-positive the expression $(\varphi(u)-\varphi(\hat{u}))^{+} \Delta \rho$ in the $\mathcal{D}^{\prime}$ formulation of (2). To this end, any positive super-harmonic vanishing at infinity function $\rho$ could be used. Among the radial super-harmonic functions, the use of the truncated fundamental solution is optimal with respect to the restrictions on $F$ that 
come from the second term in (2); indeed, the second term in (2) has to be controlled as well.

Our technique does not allow to benefit from the anisotropy of the flux $F$; in particular, unlike in [15], our assumptions do not reduce to (3) in the trivial case $\varphi \equiv 0$. The case of an anisotropic diffusion of the form $\sum_{i=1}^{N} \partial_{x_{i} x_{i}} \varphi_{i}(u)$ cannot be covered neither (except when it reduces to the isotropic case by a change of coordinates in $\mathbb{R}^{N}$; this happens when the functions $\varphi_{i}$ are multiples of some $\varphi$ ). Indeed, the anisotropy makes it impossible to benefit from the compensations between the different derivatives $\rho_{x_{i} x_{i}}$. We refer to Maliki and Ouedraogo [1] for results on the non-isotropic diffusion case under suitable assumptions on the moduli of continuity of $\varphi_{i}$, along the guidelines of [15]. The problem where the quasilinear diffusion operator $\Delta \varphi(u)$ is replaced with a fully nonlinear diffusion operator such as the $p$-Laplacian $\Delta_{p} \varphi(u)$ is even more difficult; to our knowledge, no uniqueness result for entropy solutions in $\mathbb{R}^{N}$ is available when $p>2$.

\section{Definitions and results}

Recall that $\varphi$ is a continuous function on $\mathbb{R}$. For $M>0$, we say that a sub-additive, continuous function $\omega_{\varphi}:[0,2 M] \mapsto \mathbb{R}^{+}$such that $\omega_{\varphi}(0)=0$ is a modulus of continuity of $\varphi$, if

$$
\forall z, \hat{z} \in[-M, M] \quad|\varphi(z)-\varphi(\hat{z})| \leq \omega_{\varphi}(|z-\hat{z}|) .
$$

Recall that we only work with bounded solutions of (1). Without loss of generality, we can assume that $M$ is fixed, moreover, $\omega_{\varphi}$ is strictly concave (and thus, strictly increasing) and extended to the whole of $\mathbb{R}^{+}$; we denote its inverse function by $\Omega_{\varphi}$. We use the Fenchel (Legendre) transform of $\Omega_{\varphi}$ :

$$
\Omega_{\varphi}^{*}(t)=\sup _{z \in \mathbb{R}^{+}}\left(z t-\Omega_{\varphi}(z)\right)
$$

It easily follows that $\Omega_{\varphi}^{*}$ is strictly increasing, thus it is invertible. With the above notation, we have for all $z, t, r \in \mathbb{R}^{+}$,

$$
z t \leq \Omega_{\varphi}(z)+\Omega_{\varphi}^{*}(t), \quad\left(\Omega_{\varphi} \circ \omega_{\varphi}\right)(r)=r .
$$

For a vector-valued function $F: \mathbb{R} \mapsto \mathbb{R}^{N}$, we introduce the (isotropic) modulus of continuity $\omega_{F}$ and the related functions $\Omega_{F}, \Omega_{F}^{*}$ in the same way, replacing $|\varphi(z)-\varphi(\hat{z})|$ in (4) with $|F(z)-F(\hat{z})|$. Here and in the sequel, we mean that $\mathbb{R}^{N}$ is endowed with the euclidean norm, which we denote by $|\cdot|$.

Definition 1. An entropy solution of (1) is a function $u \in L^{\infty}\left((0, T) \times \mathbb{R}^{N}\right)$ such that $\varphi(u) \in L^{2}\left(0, T ; H_{\text {loc }}^{1}\left(\mathbb{R}^{N}\right)\right)$ and the inequalities 


$$
\begin{aligned}
& -\int_{0}^{T} \int_{\mathbb{R}^{N}}\left(|u-k| \xi_{t}+\operatorname{sign}(u-k)(F(u)-F(k)) \cdot \nabla \xi\right. \\
& \quad+\operatorname{sign}(u-k) \nabla \varphi(u) \cdot \nabla \xi) \\
& \leq \int_{0}^{T} \int_{\mathbb{R}^{N}} \operatorname{sign}(u-k) f \xi+\int_{\mathbb{R}^{N}}\left|u_{0}-k\right| \xi(0, \cdot)
\end{aligned}
$$

hold for all $k \in \mathbb{R}$ and all $\xi \in \mathcal{D}\left([0, T) \times \mathbb{R}^{N}\right)$.

Here and in the sequel, we mean that $u_{0} \in L^{\infty}\left(\mathbb{R}^{N}\right),\|f(t, \cdot)\|_{L^{\infty}} \in L^{1}(0, T)$. It is clear that an entropy solution is also a solution in the sense of distributions; it suffices to use (6) with $k= \pm\|u\|_{L^{\infty}}$. It follows that in (6), one can replace sign $(u-k)$ and $|u-k|$ with $\operatorname{sign}^{+}(u-k)$ and $(u-k)^{+}$, respectively, where $\operatorname{sign}^{+}(r):=\max \{\operatorname{sign} r, 0\}$ and $r^{+}:=\max \{r, 0\}$.

Existence of an entropy solution is standard; it can be obtained, e.g., by a regularization of $F$ and $\varphi$ (when $\varphi \equiv 0$, one takes $\varphi_{\varepsilon}(z)=\varepsilon z$, which is the so-called "vanishing viscosity" approximation), with the help of the translation invariance in $x$ of the left-hand side of (1) and the $L^{1}$ contraction result of Maliki and Touré [15].

Definition 1 implies the Kato inequality (2), with the help of the doubling-of-variables approach of Kruzhkov [9] and the techniques of Carrillo [8] and Blanchard and Porretta [7] (see e.g. [3,4,15]). Inequality (2) can also be obtained without doubling the variables, with the help of the so-called kinetic formulation (see Perthame [17] for the case of a conservation law). Uniqueness of an entropy solution of (1) (and, more generally, the $L^{1}$ contraction and comparison principle) follows whenever we can pass to the limit in the Kato inequality (2) with test functions $\xi \rightarrow 1$ on $\mathbb{R}^{N}$.

We assume that $N \geq 3$ (for $N \leq 2$, the results of Maliki and Touré [15] apply with no restriction on $\varphi$ ).

Theorem 2. Let $N \geq 3$. Consider an arbitrary non-decreasing continuous function $\varphi: \mathbb{R} \mapsto \mathbb{R}$. Consider a locally Hölder continuous of order $\left(1-\frac{1}{N}\right)$ function $F: \mathbb{R} \mapsto \mathbb{R}^{N}$.

Then whenever $\left(u_{0}-\hat{u}_{0}\right)^{+} \in L^{1}\left(\mathbb{R}^{N}\right),(f-\hat{f})^{+} \in L^{1}\left((0, T) \times \mathbb{R}^{N}\right)$,

$$
\int_{\mathbb{R}^{N}}(u-\hat{u})^{+}(t, \cdot) \leq \int_{\mathbb{R}^{N}}\left(u_{0}-\hat{u}_{0}\right)^{+}+\int_{0}^{t} \int_{\mathbb{R}^{N}}(f-\hat{f})^{+} \quad \text { a.e. on }(0, T),
$$

where $u, \hat{u}$ are entropy solutions corresponding, respectively, to given initial data $u_{0}, \hat{u}_{0} \in L^{\infty}\left(\mathbb{R}^{N}\right)$ and sources $f, \hat{f}$ with $\|f(t, \cdot)\|_{L^{\infty}(\mathbb{R})},\|\hat{f}(t, \cdot)\|_{L^{\infty}(\mathbb{R})} \in$ $L^{1}(0, T)$.

In particular, under the above assumptions on $\varphi, F$ there exists a unique entropy solution $u_{u_{0}, f}$ for given data $u_{0}, f$; the map $\left(u_{0}, f\right) \mapsto u_{u_{0}, f}$ is order-preserving, and the map $u_{0} \in L^{\infty}\left(\mathbb{R}^{N}\right) \cap L^{1}\left(\mathbb{R}^{N}\right) \mapsto u_{u_{0}, 0}$ is a T-contraction in $L^{1}$. 


\section{Proof of Theorem 2}

The technique of the proof of the theorem is closely related to the previous work [3]. Roughly speaking, we take in the Kato inequality (2) radial test functions $\rho$ such that $\Delta \rho \leq 0$. This allows us to drop the second term in the right-hand side of (2); thus restrictions on $\varphi$ are bypassed. The test functions are constructed by approximation and truncation of the Laplacian's fundamental solution profile $\rho(x)=\frac{1}{|x|^{N-2}}$; this choice is explained in the introduction.

We are intended to use $(2)$ with the test functions $\rho_{\varepsilon}(x)=\left(\max \left\{\frac{|x|}{R}\right.\right.$, $1\})^{2-N-\varepsilon}$ with $\varepsilon>0, R>1$ (in order to lighten the notation, we do not indicate explicitly that $\rho_{\varepsilon}$ also depends on $R$ ). Then we let $\varepsilon \rightarrow 0$, and finally we let $R \rightarrow+\infty$.

To this end, let

$$
\rho_{\varepsilon, L}(x)=R^{N-2+\varepsilon}\left((\max \{|x|, R\})^{2-N-\varepsilon}-(L)^{2-N-\varepsilon}\right)^{+}, \quad L>R .
$$

By a density argument, we take in (2) test functions under the form $\xi(t, x)=$ $\mu(t) \rho_{\varepsilon, L}(x)$, where $\mu \in \mathcal{D}([0, T))^{+}$. Indeed, regularizing $\rho_{\varepsilon, L}$ by convolution, dropping the negative measure part of the distribution $\Delta \rho_{\varepsilon, L}$ concentrated on the set $\left\{x \in \mathbb{R}^{N}|| x \mid=R\right\}$, using the definition of $\omega_{\varphi}, \omega_{F}$ we deduce

$$
\begin{aligned}
& \int_{0}^{T} \int_{\mathbb{R}^{N}}(u-\hat{u})^{+} \rho_{\varepsilon, L}\left(-\mu_{t}\right) \\
& \leq \quad \int_{0}^{T} \int_{\mathbb{R}^{N}} \omega_{F}\left((u-\hat{u})^{+}\right)\left|\nabla \rho_{\varepsilon, L}\right| \mu+\int_{0}^{T} \int_{\mathbb{R}^{N}} \omega_{\varphi}\left((u-\hat{u})^{+}\right) \Delta^{\mathrm{ac}} \rho_{\varepsilon, L} \mu \\
& \quad+\int_{\{|x|=L\}} \omega_{\varphi}\left((u-\hat{u})^{+}\right)(N-2+\varepsilon) L^{1-N-\varepsilon} \mu \\
& \quad+\int_{0}^{T} \int_{\mathbb{R}^{N}}(f-\hat{f}) \operatorname{sign}^{+}(u-\hat{u}) \rho_{\varepsilon, L} \mu+\int_{\mathbb{R}^{N}}\left(u_{0}-\hat{u}_{0}\right)^{+} \rho_{\varepsilon, L} \mu(0)
\end{aligned}
$$

for a.e. $L>R^{\prime}$; here $\Delta^{\text {ac }} \rho_{\varepsilon, L}$ represents the absolutely continuous (with respect to the Lebesgue measure on $\mathbb{R}^{N}$ ) part of the measure $\Delta \rho_{\varepsilon, L}$. The integral on the sphere $\{|x|=L\}$ is understood with respect to the $(N-1)$-dimensional Hausdorff measure. Because $u, \hat{u}$ are bounded, the integral on the sphere $\{|x|=$ $L\}$ is upper bounded by const $L^{N-1} L^{1-N-\varepsilon}=$ const $L^{-\varepsilon}$. We calculate

$$
\Delta^{\mathrm{ac}} \rho_{\varepsilon, L}=\varepsilon(N-2+\varepsilon)|x|^{-2} \rho_{\varepsilon} \mathbb{1}_{R<|x|<L}
$$

and

$$
\left|\nabla \rho_{\varepsilon, L}\right|=(N-2+\varepsilon)|x|^{-1} \rho_{\varepsilon} \mathbb{1}_{R<|x|<L}
$$

here $\rho_{\varepsilon}(x)=\left(\max \left\{\frac{|x|}{R}, 1\right\}\right)^{2-N-\varepsilon}$. Notice that $0 \leq \rho_{\varepsilon, L} \leq 1$ for all $\varepsilon, R, L$. Now we let $L \rightarrow \infty$. Thanks to the boundedness of $u, \hat{u}$ and to the integrability 
assumption on $\left(u_{0}-\hat{u}_{0}\right)^{+},(f-\hat{f})^{+}$we deduce that

$$
\begin{aligned}
& \int_{0}^{T} \int_{\mathbb{R}^{N}}(u-\hat{u})^{+} \rho_{\varepsilon}\left(-\mu_{t}\right) \\
& \leq c \int_{0}^{T} \int_{\{|x|>R\}}\left(\omega_{F}\left((u-\hat{u})^{+}\right)|x|^{-1}+\varepsilon \omega_{\varphi}\left((u-\hat{u})^{+}\right)|x|^{-2}\right) \rho_{\varepsilon} \\
& \quad+\int_{0}^{T} \int_{\mathbb{R}^{N}}(f-\hat{f}) \operatorname{sign}^{+}(u-\hat{u}) \mu+\int_{\mathbb{R}^{N}}\left(u_{0}-\hat{u}_{0}\right)^{+} \mu(0) .
\end{aligned}
$$

Here and in the sequel, we assume $\varepsilon \in(0,1)$, so that $(N-2+\varepsilon) \leq(N-1)$, and fix $c=(N-1)\|\mu\|_{L^{\infty}}$. Notice that each term in the right-hand side of (8) is finite.

Our goal is to show that, letting $\varepsilon \rightarrow 0$ and $R \rightarrow \infty$, we can drop the first term in the right-hand side of $(8)$. The first step is to prove that $(u-\hat{u})^{+}$ is integrable on $(0, T) \times \mathbb{R}^{N}$.

To this end, fix $\delta>0$. Let us show that

$$
c \varepsilon \int_{0}^{T} \int_{\{|x|>R\}} \omega_{\varphi}\left((u-\hat{u})^{+}\right)|x|^{-2} \rho_{\varepsilon} \leq \delta \int_{0}^{T} \int_{\{|x|>R\}}(u-\hat{u})^{+} \rho_{\varepsilon}+r^{\delta, R}(\varepsilon),
$$

where for all fixed $\delta>0, R>1$, the quantity $r^{\delta, R}(\varepsilon)$ tends to zero as $\varepsilon$ decreases to zero. Indeed, using (5), we get

$$
\begin{aligned}
c \varepsilon \omega_{\varphi}\left((u-\hat{u})^{+}\right)|x|^{-2} & =\delta\left(\omega_{\varphi}\left((u-\hat{u})^{+}\right) \frac{c \varepsilon}{\delta}|x|^{-2}\right) \\
& \leq \delta(u-\hat{u})^{+}+\delta \Omega_{\varphi}^{*}\left(\frac{c \varepsilon}{\delta}|x|^{-2}\right)
\end{aligned}
$$

thus (9) holds with

$$
r^{\delta, R}(\varepsilon):=T \delta \int_{\{|x|>R\}} \Omega_{\varphi}^{*}\left(\frac{c \varepsilon}{\delta}|x|^{-2}\right) \rho_{\varepsilon} .
$$

By the convexity of $\Omega_{\varphi}^{*}$, we have $\Omega_{\varphi}^{*}\left(\frac{c \varepsilon}{\delta}|x|^{-2}\right) \leq\left(\Omega_{\varphi}^{*}\right)^{\prime}\left(\frac{c \varepsilon}{\delta} R^{-2}\right) \frac{c \varepsilon}{\delta}|x|^{-2}$ whenever $|x| \geq R$. Without loss of generality, we can assume that $\Omega_{\varphi}$ is convex and strictly increasing; then it is not difficult to prove that ess $\lim _{t \downarrow 0}\left(\Omega_{\varphi}^{*}\right)^{\prime}(t)=0$ (see e.g. [3]). Hence we deduce that $\left(\Omega_{\varphi}^{*}\right)^{\prime}\left(\frac{c \varepsilon}{\delta} R^{-2}\right)=: \overline{\bar{o}}_{\varepsilon}$ tends to zero as $\varepsilon \rightarrow 0$. Therefore using polar coordinates, we get

$$
\begin{aligned}
0 \leq r^{\delta, R}(\varepsilon) & =\delta T \int_{\{|x|>R\}} \Omega_{\varphi}^{*}\left(\frac{c \varepsilon}{\delta}|x|^{-2}\right) \rho_{\varepsilon} \leq \overline{\bar{o}}_{\varepsilon} c T \varepsilon \int_{\{|x|>R\}}|x|^{-2} \rho_{\varepsilon} \\
& =c T \varepsilon R^{N-2+\varepsilon} \overline{\bar{o}}_{\varepsilon} \int_{R}^{+\infty} r^{-2+2-N-\varepsilon} r^{N-1} d r \\
& =c T \varepsilon R^{N-2+\varepsilon} \frac{R^{-\varepsilon}}{\varepsilon} \overline{\bar{o}}_{\varepsilon} \rightarrow 0 \quad \text { as } \varepsilon \rightarrow 0 .
\end{aligned}
$$

Similarly, from (5) we deduce

$$
c \omega_{F}\left((u-\hat{u})^{+}\right)|x|^{-1} \rho_{\varepsilon} \leq \frac{1}{2}(u-\hat{u})^{+} \rho_{\varepsilon}+\frac{1}{2} \Omega_{F}^{*}\left(2 c|x|^{-1}\right) \rho_{\varepsilon} .
$$


By the Hölder continuity assumption on $F$, we can take

$$
\omega_{F}(r)=C r^{1-\frac{1}{N}} \text {, thus } \Omega_{F}(z)=C z^{\frac{N}{N-1}} \text {, thus } \Omega_{F}^{*}(t)=C t^{N} .
$$

Here $C$ is a generic constant that changes from one expression to another. Hence

$$
\int_{0}^{T} \int_{\{|x|>R\}} \Omega_{F}^{*}\left(2 c|x|^{-1}\right) \rho_{\varepsilon}=C T R^{N-2+\varepsilon} \int_{R}^{+\infty}\left(\frac{2 c}{r}\right)^{N} r^{1-\varepsilon} d r \leq \text { const. }
$$

By density, we can take $\mu(\cdot)=(T-\cdot)^{+}$in $(8)$, so that $-\mu_{t}(t)=\mathbb{1}_{(0, T)}(t)$. Putting $\delta=1 / 2$ and letting $\varepsilon \rightarrow 0$, from (8) and (9)-(13) we deduce that

$\int_{0}^{T} \int_{\{|x|<R\}}(u-\hat{u})^{+} \leq T \int_{\mathbb{R}^{N}}\left(u_{0}-\hat{u}_{0}\right)^{+}+T \int_{0}^{T} \int_{\mathbb{R}^{N}}(f-\hat{f})^{+}+$const $\leq$const.

Letting $R \rightarrow \infty$, by the monotone convergence theorem we deduce that

$$
(u-\hat{u})^{+} \in L^{1}\left((0, T) \times \mathbb{R}^{N}\right) .
$$

In order to conclude the proof, we return to (8). We now replace (11) with the estimate

$$
\begin{aligned}
c \omega_{F}\left((u-\hat{u})^{+}\right)|x|^{-1} \rho_{\varepsilon} & \leq \delta c \Omega_{F}\left(\frac{1}{\delta} \omega_{F}\left((u-\hat{u})^{+}\right)\right) \rho_{\varepsilon}+\delta c \Omega_{F}^{*}\left(|x|^{-1}\right) \rho_{\varepsilon} \\
& =\delta^{1-\frac{N}{N-1}} c C(u-\hat{u})^{+}+\delta c C|x|^{-N}\left(\frac{R}{|x|}\right)^{N-2+\varepsilon} ;
\end{aligned}
$$

the expressions (12) are used in (15). Hence setting $C(\delta)=\delta^{1-\frac{N}{N-1}} c C$ we infer

$$
\begin{aligned}
& c \int_{0}^{T} \int_{\{|x|>R\}} \omega_{F}\left((u-\hat{u})^{+}\right)|x|^{-1} \rho_{\varepsilon} \\
& \quad \leq C(\delta) \int_{0}^{T} \int_{\{|x|>R\}}(u-\hat{u})^{+}+\delta c C T R^{N-2+\varepsilon} \int_{\{|x|>R\}}|x|^{2-2 N+\varepsilon} \rho_{\varepsilon} \\
& \quad \leq C(\delta) \int_{0}^{T} \int_{\{|x|>R\}}(u-\hat{u})^{+}+\text {const } \delta .
\end{aligned}
$$

The left-hand side of (16) converges to zero as $R \rightarrow \infty$, uniformly in $\varepsilon$; indeed, (14) yields

$$
\inf _{\delta>0}\left(C(\delta) \int_{0}^{T} \int_{\{|x|>R\}}(u-\hat{u})^{+}+\text {const } \delta\right) \rightarrow 0 \quad \text { as } R \rightarrow \infty .
$$

Using (9) and (10) once more, from (14) we get

$$
\begin{aligned}
& c \varepsilon \int_{0}^{T} \int_{\{|x|>R\}} \omega_{\varphi}\left((u-\hat{u})^{+}\right)|x|^{-2} \rho_{\varepsilon} \\
& \quad \leq \inf _{\delta>0}\left(\delta \int_{0}^{T} \int_{\{|x|>R\}}(u-\hat{u})^{+}+r^{\delta, R}(\varepsilon)\right) \longrightarrow 0
\end{aligned}
$$


as $\varepsilon \rightarrow 0$ and then $R \rightarrow \infty$. Now we let $\varepsilon \rightarrow 0$ and $R \rightarrow+\infty$ in (8); notice that $\rho_{\varepsilon}$ converge to 1 and remains bounded. With the help of (16), (17) and (18), we deduce

$$
\begin{aligned}
& \int_{0}^{T} \int_{\mathbb{R}^{N}}(u-\hat{u})^{+}\left(-\mu_{t}\right) \\
& \quad \leq \int_{0}^{T} \int_{\mathbb{R}^{N}}(f-\hat{f}) \operatorname{sign}^{+}(u-\hat{u}) \mu+\int_{\mathbb{R}^{N}}\left(u_{0}-\hat{u}_{0}\right)^{+} \mu(0) .
\end{aligned}
$$

The claim (7) follows readily from (19). Because the existence of an entropy solution is already known, this ends the proof of Theorem 2 .

\section{References}

[1] Maliki, M., Ouedraogo, A.: Renormalized solution for a nonlinear anisotropic degenerated parabolic equation with nonlipschitz convection and diffusion flux functions. Int. J. Evol. Equ. 4(1) (2008)

[2] Andreianov, B., Bénilan, Ph., Kruzhkov, S.N.: $L^{1}$ theory of scalar conservation law with continuous flux function. J. Funct. Anal. 171(1), 15-33 (2000)

[3] Andreianov, B., Maliki, M.: On well-posedness for nonlinear diffusion equations in $\mathbb{R}^{N}$ (preprint)

[4] Andreu, F., Igbida, N., Mazón, J.M., Toledo, J.: Renormalized solutions for degenerate elliptic-parabolic problems with nonlinear dynamical boundary conditions and $L^{1}$-data. J. Differ. Equ. 244(11), 2764-2803 (2008)

[5] Bénilan, Ph.: Équations d'évolution dans un espace de Banach quelconque et applications. Thèse d'état (1972)

[6] Bénilan, Ph., Kruzhkov, S.N.: Conservation laws with continuous flux functions. NoDEA 3, 395-419 (1996)

[7] Blanchard, D., Porretta, A.: Stefan problems with nonlinear diffusion and convection. J. Differ. Eq. 210(2), 383-428 (2005)

[8] Carrillo, J.: Entropy solutions for nonlinear degenerate problems. Arch. Ration. Mech. Anal. 147, 269-361 (1999)

[9] Kruzhkov, S.N.: Generalized solutions of the Cauchy problem in the large for first-order nonlinear equations. Dokl.Akad.Nauk URSS V.187(1969), no. 1, pp. 29-32 (Russian); English tr. in Soviet Math.Dokl. V.10 (1969)

[10] Kruzhkov, S.N.: Conservation laws with the infinite domain of dependence on initial data. In: Barbu, V. (ed.) Differential equations and control theory. Pitman Research Notes in Mathematics, vol. 250 (1991), pp. 149-162

[11] Kruzhkov, S.N., Andrejanov, P.A.: On the nonlocal theory of the Cauchy problem for quasi-linear equations of first order in the class of locally summable functions. (English. Russian original) Sov. Math. Dokl., 16(1975), pp. 16-20; translation from Dokl. Akad. Nauk SSSR 220(1975), pp. 23-26 
[12] Kruzhkov, S.N., Hil'debrand, F.: The Cauchy problem for first order quasilinear equations in the case when the domain of dependence on the initial data is infinite. (Russian). Collection of articles dedicated to the memory of I.G. Petrovskii. Vestnik Moskov. Univ. Ser. I Mat. Meh., 29(1), 93-100 (1974)

[13] Kruzhkov, S.N., Panov, E.Yu.: First-order quasilinear conservation laws with infinite initial data dependence area. Dokl.Akad.Nauk URSS 314(1), 79-84 (1990) (Russian); English tr. in Soviet Math.Dokl. V.42(2), 316-321 (1991)

[14] Kruzhkov, S.N., Panov, E.Yu.: Osgood's type conditions for uniqueness of entropy solutions to Cauchy problem for quasilinear conservation laws of the first order. Ann. Univ. Ferrara 15, 31-53 (1994)

[15] Maliki, M., Touré, H.: Uniqueness of entropy solutions for nonlinear degenerate parabolic problem. J. Evol. Equ. 3(4), 603-622 (2003)

[16] Panov, E.Yu.: Generalized solutions of the Cauchy problem for quasilinear conservation laws. PhD thesis, Moscow State University (1991)

[17] Perthame, B.: Uniqueness and error estimates in first order quasilinear conservation laws via the kinetic entropy defect measure. J. Math. Pures Appl. (9) 77(10), 1055-1064 (1998)

B. Andreianov

Laboratoire de Mathématiques

Université de Franche-Comté

16 route de Gray

25030 Besançon Cedex, France

e-mail: boris.andreianov@univ-fcomte.fr

M. Maliki

Équipe Modélisation, EDP et Analyse Numérique

FST Mohammédia

B.P. 146, Mohammédia,

Morocco

e-mail: mohamedmaliki@yahoo.fr

Received: 30 April 2009.

Accepted: 24 September 2009. 